\title{
Spin dynamics of molecular nanomagnets unravelled at atomic scale by four-dimensional inelastic neutron scattering
}

\author{
Michael L. Baker ${ }^{1,2}$, Tatiana Guidi ${ }^{3}$, Stefano Carretta ${ }^{4}$, Jacques Ollivier ${ }^{1}$, Hannu Mutka ${ }^{1}$, \\ Hans U. Güdel ${ }^{5}$, Grigore A. Timco ${ }^{2}$, Eric J. L. Mclnnes ${ }^{2}$, Giuseppe Amoretti ${ }^{4}$, Richard E. P. Winpenny ${ }^{2}$ \\ and Paolo Santini ${ }^{4 \star}$
}

\begin{abstract}
Molecular nanomagnets are among the first examples of finite-size spin systems and have been test beds for addressing several phenomena in quantum dynamics. In fact, for short-enough timescales the spin wavefunctions evolve coherently according to an appropriate spin Hamiltonian, which can be engineered to meet specific requirements. Unfortunately, so far it has been impossible to determine these spin dynamics directly. Here we show that recently developed instrumentation yields the four-dimensional inelastic-neutron scattering function in vast portions of reciprocal space and enables the spin dynamics to be determined directly. We use the $\mathrm{Cr}_{8}$ antiferromagnetic ring as a benchmark to demonstrate the potential of this approach which allows us, for example, to examine how quantum fluctuations propagate along the ring or to test the degree of validity of the Néel-vector-tunnelling framework.
\end{abstract}

M agnetic nanostructures are promising components of emergent technologies in spintronics ${ }^{1-7}$ and quantuminformation processing ${ }^{8,9}$. The finite size of these clusters can result in typical quantum phenomena, such as tunnelling through an energy barrier or coherent oscillations associated with superpositions of states. Tailored nanomagnets can be assembled on a surface by using a scanning tunnelling microscope $\mathrm{e}^{10-12}$ or can be produced in the form of metallorganic molecules whose magnetic cores are isolated by shells of ligands and behave as zerodimensional units ${ }^{13}$. In these molecular nanomagnets (MNMs), the structure of the cores and the topology of exchange interactions can be controlled precisely at the chemical synthesis level. MNMs can be grafted onto surfaces without significantly perturbing their properties $^{2,14,15}$, and have the advantage with respect to other nanostructures that a macroscopic number of identical independent units can be gathered in the form of crystals of exceedingly high quality. These enable single-molecule properties to be finely explored by bulk techniques, making MNMs test beds for fundamental concepts in quantum mechanics such as quantum-tunnelling of the magnetization ${ }^{16,17}$, Berry-phase interference ${ }^{18}$, frustration ${ }^{19}$, Néel-vector tunnelling ${ }^{20,21}$ (NVT), quantum information ${ }^{8,9,22-24}$, entanglement ${ }^{25-28}$ or decoherence ${ }^{29-32}$.

A crucial aspect of the research on MNMs is the understanding of their spin dynamics, especially of those aspects being a direct manifestation of quantum mechanics such as the tunnelling of the Néel-vector in antiferromagnetic rings ${ }^{13}$. For short-enough timescales (for example, less than some microseconds in rings ${ }^{29,32}$ ), the molecular wavefunctions evolve coherently according to the Schrödinger equation for an appropriate spin Hamiltonian. It is difficult to probe this evolution directly because of its small characteristic length- and timescales, typically of the order of a few angströms and $0.1 \mathrm{ps}$, respectively. The most powerful technique to investigate the spin dynamics in bulk samples is inelastic neutron scattering (INS). In MNMs, the dynamics are usually extrapolated by fitting INS spectra to a spin Hamiltonian and by performing calculations within the framework set by this model. However, this indirect approach does not work in many interesting cases when the Hilbert space dimension is too large or when a large number of model parameters makes the fit non-univocal. Here we show that measurements of the scattering function over a large solid angle of wave vectors in high-quality single crystals of MNMs permit us to directly determine real-space dynamical two-spin correlations without assuming any underlying model Hamiltonian. These correlations are the key quantities characterizing the magnetic dynamics. For instance, they determine the linear response of the system to an arbitrary magnetic field varying in space and time. We exploit $\mathrm{Cr}_{8}$, a prototype antiferromagnetic ring ${ }^{33}$, as a benchmark to demonstrate the potential of this approach for the determination of the spin dynamics in MNMs. The so-obtained correlation functions allow a model-free picture of the quantum dynamics of the ring. For example, the way a quantum fluctuation propagates along the ring or the degree of validity of a NVT description $^{20,21,34}$ are determined.

\section{The $\mathrm{Cr}_{8}$ prototype antiferromagnetic ring}

The $\mathrm{Cr}_{8}$ molecule contains eight $\mathrm{Cr}^{3+}$ ions $(s=3 / 2)$ forming a nearly regular octagon ${ }^{33,35}$. It is one of the most studied MNMs, both for its intrinsic interest and for being the precursor of a large family of heterometallic molecules and supramolecular complexes that are interesting for a number of fundamental and applicative

\footnotetext{
${ }^{1}$ Institut Laue-Langevin, BP 156, 6 rue Jules Horowitz, 38042 Grenoble Cedex 9, France, ${ }^{2}$ School of Chemistry and Photon Institute, The University of Manchester, Manchester M13 9PL, UK, ${ }^{3}$ ISIS facility, Rutherford Appleton Laboratory, Didcot OX11 OQX, UK, ${ }^{4}$ Dipartimento di Fisica e Scienze della Terra, Università di Parma, I-43124 Parma, Italy, ${ }^{5}$ Department of Chemistry and Biochemistry, University of Bern, 3000 Bern, Switzerland. *e-mail:paolo.santini@unipr.it.
} 
issues ${ }^{9,27,28,36,37}$. The spin Hamiltonian of $\mathrm{Cr}_{8}$ contains a dominating nearest-neighbour Heisenberg antiferromagnetic exchange and small axial anisotropic terms:

$$
H=J \sum_{i=1}^{8} \mathbf{s}(i) \cdot \mathbf{s}(i+1)+D \sum_{i=1}^{8} s_{z}^{2}(i)
$$

where $\mathbf{s}(9) \equiv \mathbf{s}(1), J=1.46 \mathrm{meV}, D=-0.038 \mathrm{meV}$ and the anisotropy axis $z$ is perpendicular to the ring plane $e^{35,38}$. The ground state of equation (1) is a non-magnetic singlet (total spin $S=0$ ), and the low-lying excited levels are arranged into rotational bands (Fig. 1). The lowest one (called the L-band) contains the ground state and approximately follows the Landé rule $E(S)=2 J S(S+1) / N$ (ref. 39). The second set of levels belongs to the so-called E-bands, which are also parabolic with respect to $S$ but shifted to higher energies. Owing to their internal structure, L-band states can be excited by neutrons practically only to L- or E-band states ${ }^{40}$. Hence, at low temperature, where only the $S=0$ ground state is populated, three peaks are expected and observed in INS spectra ${ }^{35}$ (transitions marked by arrows in Fig. 1). Anisotropy produces small splittings of the otherwise degenerate $S$-multiplets and a tiny second-order mixing of different multiplets. For instance, the L-band $S=1$ triplet is split into an $|S=1, M=0\rangle$ singlet and an $|S=1, M= \pm 1\rangle$ doublet that are resolved by high-resolution INS measurements (inset of Fig. 1b). Splittings of the E-band triplets are smaller and not experimentally resolved.

\section{INS and dynamical correlation functions}

The INS spectra in Fig. 1b have been obtained on a polycrystalline sample and are integrated over a wide range of momentum transfer Q; hence, they contain no direct information on the spatial structure of wavefunctions. This is usually indirectly inferred by fitting the spectra to a specific model Hamiltonian such as equation (1) or by analysing the dependence of peak intensities on the modulus of the momentum transfer $Q$. In powder INS measurements, however, the resulting angular-averaged cross-section does not in general allow one to extract dynamical spin correlations directly. Indeed, in the corresponding cross-section formula (see refs 34,41) all anisotropic correlation coefficients multiply the same $Q$-dependent function and therefore cannot be extracted separately. Even in the isotropic case, the fact that the powder cross-section depends only on scalar distances between magnetic ions and not on their vector positions in space poses severe limitations. For instance, correlations in the simple but important case of a heterometallic ring cannot be determined if only powder data are available, because this case cannot be distinguished from that of a homometallic ring with averaged correlations.

If single-crystal samples are used, only limited and partially integrated information on the $\mathbf{Q}$-dependence of the scattering function $S(\mathbf{Q}, \omega)$ (where $\hbar \omega$ is the transferred energy, see ref. 42 and equation (2)) can be obtained by traditional timeof-flight neutron spectrometers, which carry unitary detectors fitted on Debye-Scherrer rings. The implementation of large arrays of position-sensitive detectors in cold-neutron time-of-flight spectrometers $^{43,44}$, together with the advances in software ${ }^{45}$, has recently opened unprecedented possibilities in single-crystal INS experiments on MNMs, allowing the determination of the fourdimensional scattering function $S(\mathbf{Q}, \omega)$ in a vast portion of the reciprocal space. On the one hand, this provides a much more selective characterization of the MNM when different candidate models can be discriminated only by the vectorial $\mathbf{Q}$-dependence of $S(\mathbf{Q}, \omega)$ (ref. 41). On the other hand, the amount of available information is so large that the full pattern of real-space dynamical two-spin correlations can be determined, without using any model Hamiltonian.

By exploiting the new position-sensitive-detectors set-up of the cold-neutron time-of-flight spectrometer IN5 (ref. 43), we have
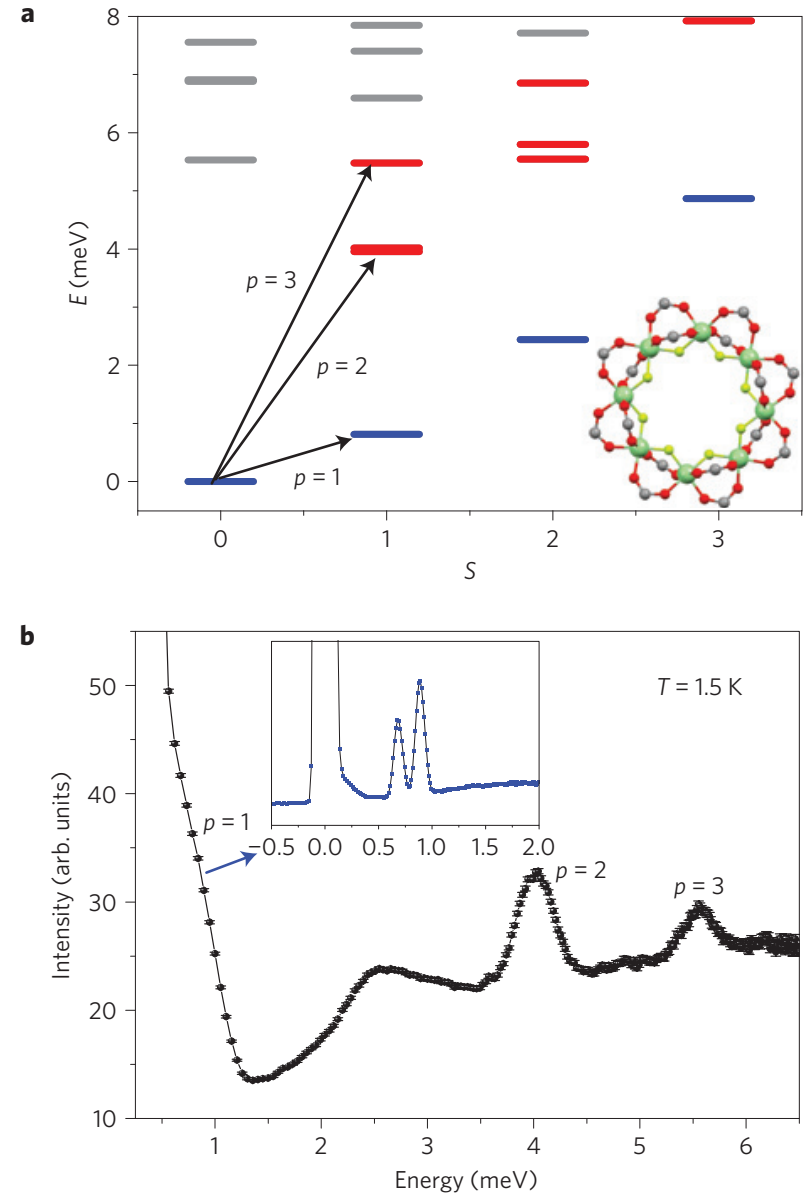

Figure 1 | Magnetic energy spectrum of $\mathrm{Cr}_{8}$ and zero-temperature INS transitions. a, Low-lying energy multiplets as a function of their total spin for the isotropic exchange Hamiltonian of $\mathrm{Cr}_{8}$ (equation (1) with $D=0$ ).

The arrows indicate the three transitions seen by INS at zero temperature. All other transitions have negligible cross-section. The blue and red symbols indicate L- and E-band states, respectively; grey symbols indicate states not belonging to these bands. The inset shows the core of $\mathrm{Cr}_{8}$ ( $\mathrm{C}_{80} \mathrm{Cr}_{8} \mathrm{~F}_{8} \mathrm{D}_{144} \mathrm{O}_{32}$; green, $\mathrm{Cr}$; yellow, $\mathrm{F}$; red, O; dark grey, $\mathrm{C}$; D omitted). b. Measured low- $T$ INS spectra for a powder $\mathrm{Cr}_{8}$ sample, with an incident neutron wavelength $\lambda=3.1 \AA$. The labels indicate the three peaks corresponding to the transitions reported in $\mathbf{a}$. The $p=1$ transition is partially hidden by the elastic signal. The inset reports higher-resolution measurements with $\lambda=5 \AA$ showing the $p=1$ transition split by magnetic anisotropy.

measured the detailed Q-dependence of the scattering function $S(\mathbf{Q}, \omega)$ in a vast portion of reciprocal space. Figure 2 and Fig. 3a-c show examples of the $\mathbf{Q}$-dependence of $S(\mathbf{Q}, \omega)$ for the three low-temperature INS transitions. As we first address the effects of isotropic exchange, data for the $p=1$ transition are integrated over the energy range of the anisotropic splitting. Anisotropy will be addressed below. Figure 3 shows that the variation of $S(\mathbf{Q}, \omega)$ in the $Q_{x}-Q_{y}$ plane is characterized by several maxima and minima whose shape and positions depend on the specific transitions and reflect the structure of the involved wavefunctions. More specifically, for a MNM with uniaxial anisotropy and $T \rightarrow 0$ (ref. 42)

$$
\begin{gathered}
S(\mathbf{Q}, \omega) \propto \sum_{\alpha=x, y, z}\left(1-\frac{Q_{\alpha}^{2}}{Q^{2}}\right) \sum_{p} \sum_{d \geq d^{\prime}=1}^{N} F_{d}(Q) \times F_{d^{\prime}}(Q) \cos \left(\mathbf{Q} \cdot \mathbf{R}_{d d^{\prime}}\right) \\
\times\left\langle 0\left|s_{\alpha}(d)\right| p\right\rangle\left\langle p\left|s_{\alpha}\left(d^{\prime}\right)\right| 0\right\rangle \delta\left(\omega-E_{p} / \hbar\right)
\end{gathered}
$$




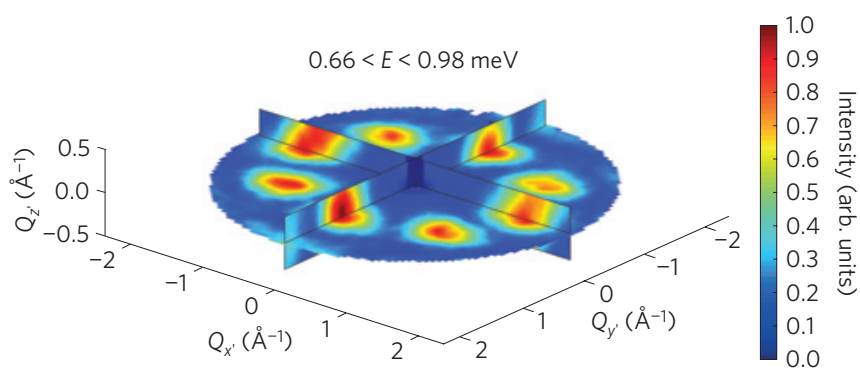

Figure 2 | Constant-energy plots of the neutron scattering intensity for the $p=1$ excitation. Cuts are shown in the $Q_{x^{\prime}}-Q_{y^{\prime}}, Q_{z^{\prime}}-Q_{y^{\prime}}$ and $Q_{z^{\prime}}-Q_{x^{\prime}}$ planes, where the primed reference frame is defined in Supplementary Fig. S1. The measurement was carried out with a $5 \AA$ incident neutron wavelength, and a sample temperature of $1.5 \mathrm{~K}$.

where $F_{d}(Q)$ is the form factor for the $d$ th ion, $|0\rangle$ and $|p\rangle$ are ground and excited eigenstates, respectively, $E_{p}$ are eigenenergies and $\mathbf{R}_{d d}$ are the relative positions of the $N$ magnetic ions within a molecule. As is well known, this formula can be rewritten in terms of $T=0$ dynamical correlation functions

$$
\left\langle s_{\alpha}(d, t) s_{\alpha}\left(d^{\prime}, 0\right)\right\rangle=\sum_{p}\left\langle 0\left|s_{\alpha}(d)\right| p\right\rangle\left\langle p\left|s_{\alpha}\left(d^{\prime}\right)\right| 0\right\rangle \mathrm{e}^{-i \omega_{p} t}
$$

where $\omega_{p}=E_{p} / \hbar$. In fact, the Fourier coefficients $c_{d d^{\prime}}^{\alpha}\left(\omega_{p}\right)=$ $\left\langle 0\left|s_{\alpha}(d)\right| p\right\rangle\left\langle p\left|s_{\alpha}\left(d^{\prime}\right)\right| 0\right\rangle$ in equation (3) coincide with the coefficients in equation (2). Whereas the values of $\omega_{p}$ are directly read out from the energies of the peaks in the INS spectrum, the $c_{d d^{\prime}}^{\alpha}\left(\omega_{p}\right)$ can be extracted from the data by fitting equation (2) to the observed Q-dependence of each peak. Indeed, for each value of $E_{p}$ these coefficients are the only unknown quantities in equation (2). To fix

the scale-factor in equation (2) we have exploited the sum rule

$$
\left\langle 0\left|s^{2}(d)\right| 0\right\rangle=s(s+1) \equiv \sum_{\alpha, p} c_{d d}^{\alpha}\left(\omega_{p}\right)
$$

with $s(s+1)=3.75$.

The Fourier coefficients $c_{d d^{\prime}}^{\alpha}\left(\omega_{p}\right)$ extracted by the fits of data integrated over the anisotropy splittings are given in Table 1. They do not depend significantly on $\alpha$ within experimental uncertainty, consistently with the first-order-perturbation effect of anisotropy in $\mathrm{Cr}_{8}$. Figure $3 \mathrm{~d}-\mathrm{f}$ shows calculated $\mathbf{Q}$-dependencies corresponding to the best-fit Fourier coefficients $c_{1 d}^{\alpha}\left(\omega_{p}\right)$. The agreement with experimental maps is excellent. The $d$-dependence of $c_{1 d}^{\alpha}\left(\omega_{p}\right)$, which determines the spatial pattern of correlations at the various frequencies, strongly varies with $\omega_{p}$. This is in line with the behaviour expected from the model of equation (1) (see Table 1).

The information on the low- $T$ spin dynamics embedded in the dynamical correlation functions (equation (3)) can be visualized by exploiting the well-known link between these correlations and linear response functions ${ }^{42}$. The building blocks of such functions are the set of susceptibilities $\chi_{\alpha, d}^{\alpha^{\prime}, d^{\prime}}(t)$, which provide the response of $s_{\alpha}(d)$ at time $t$ to a delta-pulse perturbation produced by a field $b$ of direction $\alpha^{\prime}$ applied on spin $d^{\prime}$ at time zero:

$$
\delta H(t)=-b s_{\alpha^{\prime}}\left(d^{\prime}\right) \delta(t) \rightarrow\left\langle s_{\alpha}(d)\right\rangle(t)-\left\langle s_{\alpha}(d)\right\rangle_{\mathrm{eq}}=b \chi_{\alpha, d}^{\alpha^{\prime}, d^{\prime}}(t)
$$

with

$$
\begin{aligned}
\chi_{\alpha, d}^{\alpha^{\prime}, d^{\prime}}(t) & =\frac{i}{\hbar} \Theta(t)\left\langle\left[s_{\alpha}(d, t), s_{\alpha^{\prime}}\left(d^{\prime}\right)\right]\right\rangle \\
& \equiv \delta_{\alpha, \alpha^{\prime}} \frac{2}{\hbar} \Theta(t) \sum_{p} c_{d d^{\prime}}^{\alpha}\left(\omega_{p}\right) \sin \left(\omega_{p} t\right)
\end{aligned}
$$

a

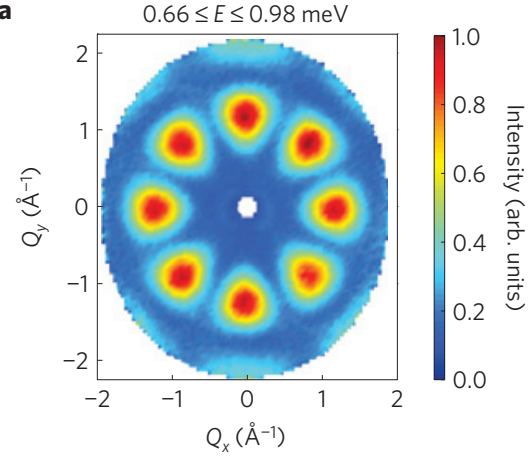

d

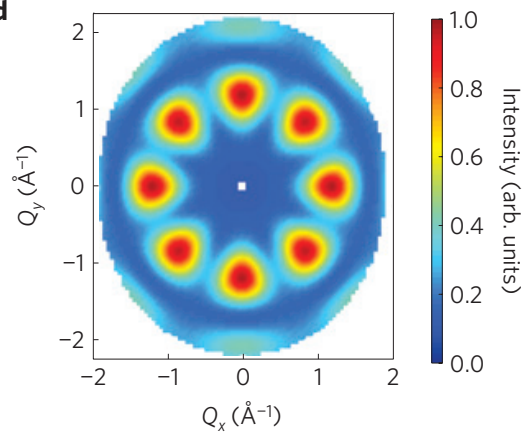

b
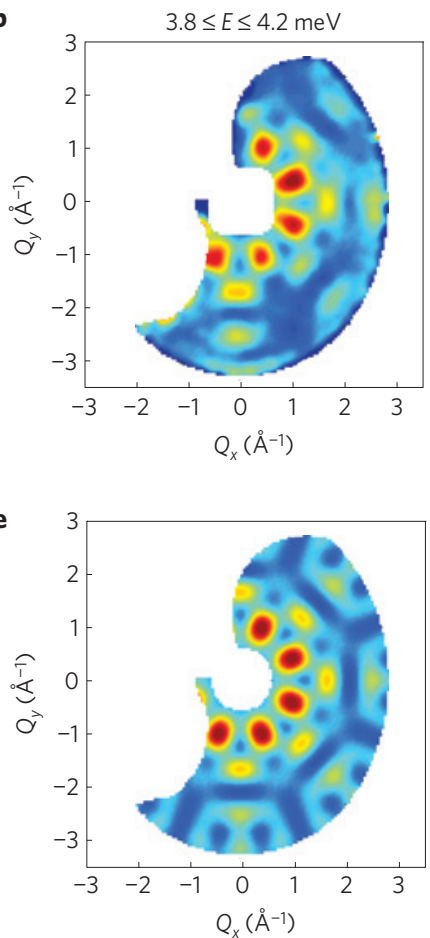

c

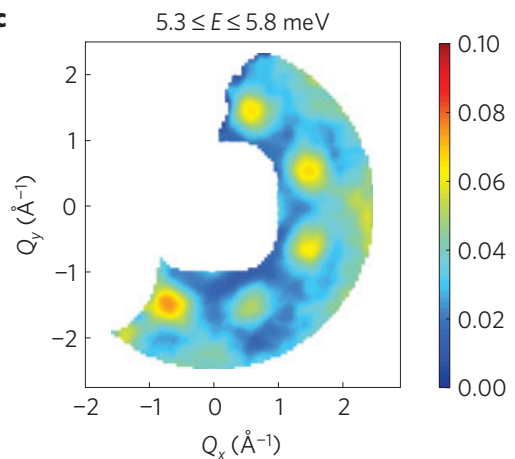

0.14

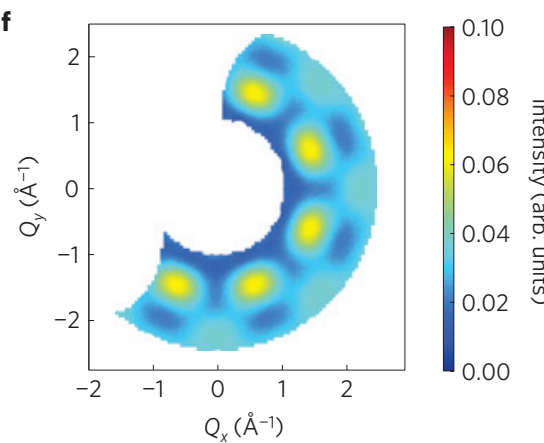

Figure 3 | Constant-energy plots of the neutron scattering intensity for all of the possible magnetic excitations in $\mathrm{Cr}_{8}$ at $1.5 \mathrm{~K}$. The maps show the dependency of intensity on two wave-vector components $Q_{x}-Q_{y}$ lying in the ring's x-y plane, integrated over the full $Q_{z}$ range. $\mathbf{a}$, Data from transition $p=1$, measured with a $5.0 \AA$ incident neutron wavelength. b,c, Data from transitions $p=2(\mathbf{b})$ and $p=3(\mathbf{c})$, measured with a $3.1 \AA$ incident neutron wavelength. d-f, Fits to equation (2) for excitations 1 (d), 2 (e) and 3 (f). One-dimensional cuts along specific directions in the $Q_{x}-Q_{y}$ plane are shown in Supplementary Fig. S2. 
Table 1 | Fourier components of two-spin dynamical correlations $c_{1 d}^{x}\left(\omega_{p}\right)=c_{1 d}^{y}\left(\omega_{p}\right)=c_{1 d}^{z}\left(\omega_{p}\right)$ extracted from the present data (Exp column), and calculated by the isotropic Heisenberg model (equation (1) with $D=0$ ).

\begin{tabular}{lrrrrrr} 
& $\boldsymbol{p}=\mathbf{1}$ & \multicolumn{3}{c}{$\boldsymbol{p = 2}$} & \multicolumn{3}{c}{$\boldsymbol{p} \mathbf{3}$} & \\
\hline & \multicolumn{1}{c}{ Exp } & Heis & Exp & Heis & Exp & Heis \\
$d=1$ & 0.78 & 0.772 & 0.28 & 0.336 & 0.19 & 0.125 \\
$d=2$ & -0.77 & -0.772 & -0.15 & -0.173 & 0.01 & 0.0 \\
$d=3$ & 0.78 & 0.772 & 0.0 & 0.0 & -0.14 & -0.125 \\
$d=4$ & -0.80 & -0.772 & 0.18 & 0.173 & -0.01 & 0.0 \\
$d=5$ & 0.81 & 0.772 & -0.32 & -0.336 & 0.1 & 0.125
\end{tabular}

The uncertainty on the experimental coefficients is of the order of 0.04 for $d>1$ and of 0.06 for $d=1$. Coefficients for $d=6,7,8$ are equivalent by symmetry to $d=4,3,2$, respectively.

$\Theta$ being the step function and $\left\langle s_{\alpha}(d)\right\rangle_{\mathrm{eq}}$ being the equilibrium average (vanishing in the present case). The response is diagonal $\left(\delta_{\alpha, \alpha^{\prime}}\right)$ because of axial symmetry. As an example, we show in Fig. 4 how a fluctuation on a given site at $t=0$ (produced by a delta-pulse field on site 1) propagates in subsequent times along the ring. The three-frequency spectrum in Table 1 produces a wave-like motion of the magnetization, which propagates forth and back along the ring with a pattern determined by the precise values of the Fourier coefficients. After the pulse is applied on site 1, waves propagating clockwise and anticlockwise interfere constructively at the opposite $(d=5)$ site, thus producing a large value of local magnetization $\left\langle s_{z}(5)\right\rangle$ after about $7 \times 10^{-13} \mathrm{~s}$. Such constructive interference is lacking in the case of an odd-numbered antiferromagnetic ring. Here, frustration would produce a complex time dependence when the two waves meet, which could be deduced by the present technique.

The measurement of the whole set of low- $T$ Fourier coefficients also allows us to extract equal-time correlation functions (that is, equation (3) with $t=0$ ), which are important quantities widely used to characterize the ground state. The advantage here is that one can isolate the weak magnetic signal by integrating over the inelastic response. Such information would be extremely challenging to obtain by other techniques such as diffuse scattering, even with polarization analysis. The staggered pattern of equal-time correlations (see Supplementary Fig. S3) has an envelope slowly decreasing with distance as expected for the one-dimensional antiferromagnetic Heisenberg model with $s=3 / 2$, which has a quantum critical ground state with power-law correlations ${ }^{46}$.
Table 2 | Fourier components of two-spin dynamical correlations $c_{1 d}^{x}\left(\omega_{p}\right)=c_{1 d}^{y}\left(\omega_{p}\right)$ and $c_{1 d}^{z}\left(\omega_{p}\right)$ extracted from the present data.

\begin{tabular}{llrrr} 
& $\mathbf{E}=\mathbf{0 . 7} \mathbf{~ m e V}$ & \multicolumn{3}{c}{$\boldsymbol{E}=\mathbf{0 . 9} \mathbf{m e V}$} \\
\hline & $x x, y y$ & $z z$ & $x x, y y$ & \multicolumn{1}{c}{$z z$} \\
$d=1$ & 0.00 & 0.82 & 0.76 & -0.02 \\
$d=2$ & 0.00 & -0.84 & -0.75 & 0.00 \\
$d=3$ & 0.00 & 0.81 & 0.72 & -0.02 \\
$d=4$ & 0.00 & -0.94 & -0.76 & 0.00 \\
$d=5$ & 0.01 & 0.95 & 0.85 & -0.06 \\
\hline
\end{tabular}

The uncertainty on the experimental coefficients is of the order of 0.05 . Calculations with the model of equation (1) yield: alternating \pm 0.86 for the $z z$ coefficients and zero for the $x x$ and $y y$ ones $(0.7 \mathrm{meV}$ peak); alternating \pm 0.73 for the $x x$ and $y y$ coefficients and zero for the $z z$ ones $(0.9 \mathrm{meV}$ peak). The average of the $x x, y y$ and $z z$ coefficients is consistent with the values for the unresolved $p=1$ transition in Table 1 .

\section{Anisotropy}

As discussed above, magnetic anisotropy in $\mathrm{Cr}_{8}$ is small and its main effect is to cause a splitting of the $S=1$ triplet into a $|M=0\rangle$ singlet and a $|M= \pm 1\rangle$ doublet, with $M$ being the magnetic quantum number. This splitting is witnessed by the presence of two separate peaks at 0.7 and $0.9 \mathrm{meV}$ in the high-resolution INS spectrum (see the inset of Fig. 1b). We have investigated the effects of magnetic anisotropy on the spin dynamics by measuring the Q-dependence of the neutron scattering intensity separately for the two low-energy peaks. The results are shown in the two upper panels of Supplementary Fig. S4. The positions of the maxima are the same for both peaks but the relative intensities of the various maxima are different. In particular, the two $Q_{x}=0$ maxima are the most intense for the $0.7 \mathrm{meV}$ transition but are the weakest for the $0.9 \mathrm{meV}$ one. We have followed the same procedure as before to fit separately the $\mathbf{Q}$-dependencies of the two peaks. The so-extracted Fourier coefficients are reported in Table 2 and the corresponding calculated maps are shown in the two lower panels of Supplementary Fig. S4. Table 2 shows that $c_{d d^{\prime}}^{\alpha}\left(\omega_{p}\right)$ are non-negligible only for $\alpha=z$ for the first peak and for $\alpha=x, y$ for the second peak. This is exactly the behaviour expected for the axial Hamiltonian of equation (1), and means that the low-frequency oscillations of $\left\langle s_{z}(d, t) s_{z}\left(d^{\prime}, 0\right)\right\rangle$ occur at a smaller frequency than those of $\left\langle s_{x}(d, t) s_{x}\left(d^{\prime}, 0\right)\right\rangle$ and $\left\langle s_{y}(d, t) s_{y}\left(d^{\prime}, 0\right)\right\rangle$. We find a slightly larger weight of the $z z$ coefficients (first peak) with respect to the $x x$ and $y y$ ones (second peak), consistent with the asymmetry expected from the model of equation (1) in the presence of $S$-mixing ${ }^{35}$.

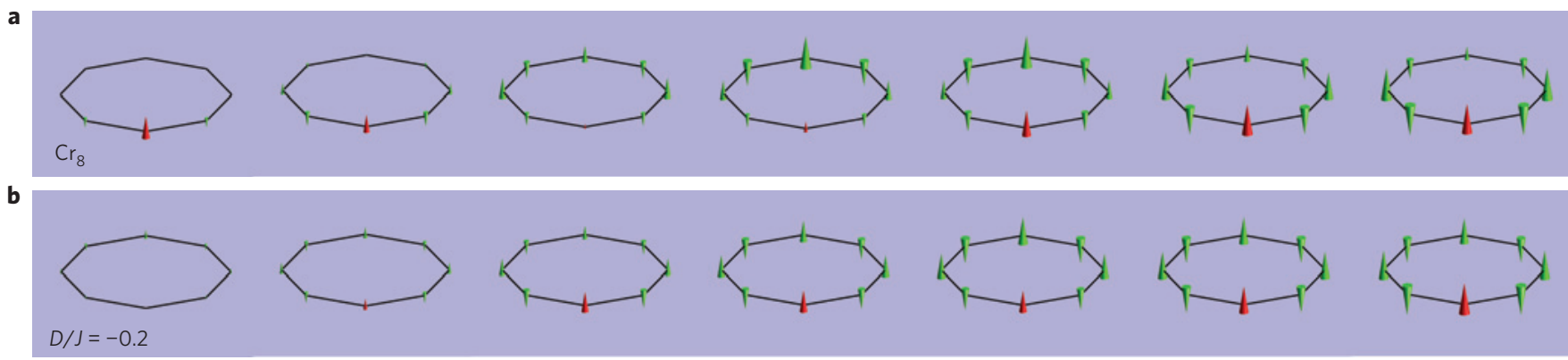

Figure 4 | Propagation of a local disturbance deduced from the present INS spectra and in a NVT regime. The frames show the time evolution of $\left\langle s_{Z}(i)\right\rangle$ in an eight-spin ring after a delta-pulse perturbation $-b s_{z}(1) \delta(t)$ applied on the red $(d=1)$ spin. Note that as the ground state is a singlet, $\left\langle s_{z}(i)\right\rangle=0$ just before $t=0$ (frame not shown). a, Dynamics deduced from equations (4) and (5) using the present experimental Fourier coefficients (Tables 1 and 2 ) and frequencies. The delay between two frames is $1.85 \times 10^{-13} \mathrm{~s}$, that is, $1 / 32$ of the longest oscillation period $2 \pi \hbar / \Delta$, with $\Delta=0.7 \mathrm{meV}$. b. Results for a hypothetical ring in a NVT regime, that is, $D / J=-0.2$ corresponding to a tunnel action $\mathcal{S}_{0} \sim 7.6$. The delay between two frames is $1 / 32$ of the tunnelling oscillation period $2 \pi \hbar / \Delta$, with $\Delta$ being the tunnelling gap. 


\section{NVT}

It has been proposed ${ }^{20}$ that the low-temperature dynamics of antiferromagnetic rings could be characterized by the coherent tunnelling of the Néel vector $\mathbf{n}=\sum_{d}(-1)^{d} \mathbf{s}(d)$ between the two classically degenerate configurations. This phenomenon is the antiferromagnetic counterpart of the tunnelling of the magnetization (quantum tunnelling of magnetization, QTM) observed in singlemolecule magnets ${ }^{13}$. Interestingly, whereas for QTM the tunnelling time is usually so long that the dynamics becomes overdamped owing to interaction with the nuclear-spins subsystem, the timescale of NVT in antiferromagnetic rings could be much shorter than the decoherence time. Within the semiclassical framework, the condition for the occurrence of NVT is that the action $S_{0}=N s \sqrt{-2 D / J}$ be much larger than unity. Hence, NVT is expected to occur in long antiferromagnetic rings with large anisotropy. Whereas QTM can be directly probed by macroscopic magnetization measurements, NVT is elusive and a direct experimental demonstration of its occurrence is lacking. NVT has recently been shown to occur in a $\mathrm{Fe}_{18}$ ring ${ }^{21}$, but only indirectly through powder INS and bulk thermodynamic measurements.

The possible occurrence of the NVT scenario in the low- $T$ dynamics of antiferromagnetic rings can be assessed by extracting dynamical correlation functions with the method presented in the paper. Indeed, in the case of NVT the two lowest eigenstates are close to the symmetric and antisymmetric superpositions of the two Néel states $|\uparrow \downarrow \uparrow \ldots\rangle$ and $|\downarrow \uparrow \downarrow \ldots\rangle$. This implies that all of the spins are rigidly locked into a two-sublattice pattern. If a local perturbing field is applied along $z$ on a single spin as discussed before, the expectation values of all the spins jointly increase in modulus (with an alternating pattern) with a practically instantaneous propagation of the perturbation. This behaviour, which already emerges for rather low $D / J$ ratios (see Fig. $4 \mathrm{~b}$ ), is completely different from the one we have experimentally found in $\mathrm{Cr}_{8}$ (Fig. 4a). Hence, these results demonstrate that the low- $T$ dynamics of $\mathrm{Cr}_{8}$ is not characterized by NVT. Figure 4 shows that even for $|D / J|<1$ (but larger than in the case of $\mathrm{Cr}_{8}$ ) NVT dynamics occurs. The present method could be applied to $\mathrm{Fe}_{18}$ to deeply investigate its spin dynamics and show that it exhibits NVT, circumventing also the fact that the huge dimension of its Hilbert space prevents exact calculations.

\section{Perspectives}

By exploiting the $\mathrm{Cr}_{8}$ prototype antiferromagnetic ring as a benchmark, we have shown that the quantum spin dynamics of MNMs can be fully unravelled by four-dimensional INS. The possibility of extracting all two-spin dynamical correlation functions opens remarkable perspectives in the understanding of crucial but elusive quantum phenomena in several classes of magnetic molecules. In particular, supramolecular complexes containing linked MNMs have been demonstrated to show entanglement ${ }^{27,28}$ and to be excellent candidates for implementing quantum-computation $^{9}$ and quantum-simulation algorithms $\mathrm{s}^{24}$. The present method could be applied to reach a profound knowledge of the structure of the eigenstates of these complexes and of the entanglement between different molecular qubits.

Another emerging field is that of frustrated MNMs (refs 19,4749), whose magnetic correlations and spin dynamics are particularly complex. In fact, their energy spectrum has a structure with large degeneracies that cannot be rationalized in terms of simple paradigms (for example, giant spins or rotational bands). However, a deep understanding of the spin dynamics of these systems is often hindered by the very large dimension of the associated Hilbert space. The approach described here can be exploited to determine dynamical spin correlations in some of these systems.

A further possible application of the method is the study of some elusive size effects in Heisenberg spin chains. For instance, in finite $S=1$ segments the valence-bond-solid structure of the spin-liquid ground state of the chain results in peculiar edge excitations of effective $s=1 / 2$ degrees of freedom. These can be studied by INS in doped bulk magnets, characterized by a statistical distribution of segment sizes ${ }^{50}$. By applying the present technique to MNMs containing magnetic arrays or open rings a targeted and precise investigation of such subtle features and their size dependence would be possible.

\section{Methods}

The original procedure for the preparation of the compound

$\mathrm{Cr}_{8} \mathrm{~F}_{8}\left[\left(\mathrm{O}_{2} \mathrm{CC}\left(\mathrm{CH}_{3}\right)_{3}\right]_{16}\left[\left(\mathrm{CH}_{3}\right)_{2} \mathrm{CO}\right]_{2}\right.$ (compound 1) reported in ref. 51, and then the modified version published in ref. 33 produce the perdeuterated version of compound 1 in a very low yield (probably caused by the isotope effects on acidity of deuterated pivalic acid) and a new synthetic procedure for the deuterated compound $\mathrm{Cr}_{8} \mathrm{~F}_{8}\left[\left(\mathrm{O}_{2} \mathrm{CC}\left(\mathrm{CD}_{3}\right)_{3}\right]_{16}\right.$ (compound 2 ) was developed and is reported below.

Heating deuterated pivalic acid $\left(\left(\mathrm{CD}_{3}\right)_{3} \mathrm{CCO}_{2} \mathrm{H}, 11.0 \mathrm{~g}, 98.94 \mathrm{mmol}\right)$, hexamethylenetetramine $\left(\mathrm{C}_{6} \mathrm{H}_{11} \mathrm{~N}_{4}, 0.6 \mathrm{~g}, 4.28 \mathrm{mmol}\right)$ and chromium(III) fluoride tetrahydrate $\left(\mathrm{CrF}_{3} \cdot 4 \mathrm{H}_{2} \mathrm{O}, 3.0 \mathrm{~g}, 16.57 \mathrm{mmol}\right)$ in a Teflon flask at $150^{\circ} \mathrm{C}$ for $14 \mathrm{~h}$ while stirring resulted in a green microcrystalline product. After this, the flask was cooled to room temperature, and acetonitrile $(20 \mathrm{ml})$ was added to complete the precipitation. The product was collected by filtration, washed with acetone, dried and then was extracted with hexane $(50 \mathrm{ml})$, and finally purified on a silica gel column using toluene as the eluent. The first fraction from the column was collected and the solution was evaporated to dryness. Yield: $3.15 \mathrm{~g} \mathrm{(65 \% ).}$ Elemental analysis calculated (\%) for $\mathrm{C}_{80} \mathrm{Cr}_{8} \mathrm{~F}_{8} \mathrm{D}_{144} \mathrm{O}_{32}$ : $\mathrm{Cr} 17.85, \mathrm{C} 41.22$; found: Cr 17.80, C 40.99. ES-MS (sample dissolved in THF; spectra run in $\mathrm{CH}_{3} \mathrm{OH}$ ) : $2352^{+}[\mathrm{M}+\mathrm{Na}]^{+}, 2,384^{+}[\mathrm{M}+\mathrm{CH} 3 \mathrm{OH}+\mathrm{Na}]^{+}$

Large crystals of $\mathrm{Cr}_{8}$ were obtained by very slow evaporation of hexane solution of compound 2 at ambient condition. The crystal structure is tetragonal (space group P4212) with $a=b=20.093(2) \AA$ and $c=16.801(2) \AA$.

INS experiments were performed on the IN5 time-of-flight inelastic neutron spectrometer ${ }^{43}$ at the high-flux reactor of the Institute Laue-Langevin. The IN5 instrument has a $30 \mathrm{~m}^{2}$ position-sensitive detector divided into $10^{5}$ pixels, covering $147^{\circ}$ of azimuthal angle and $\pm 20^{\circ}$ out-of plane. A $0.24 \mathrm{~g} \mathrm{Cr}_{8}$ single crystal was aligned as in Supplementary Fig. S1 and measurements were taken by rotating the crystal (in steps of $1^{\circ}$ ) about the vertical $z^{\prime}$ axis. Incident neutron wavelengths of 5.0 and $3.1 \AA$ were selected to probe measured excitations with energy resolutions, at zero energy transfers, of 0.079 and $0.38 \mathrm{meV}$ respectively. The uniformity of the detector sensitivity was ensured by measurement of a standard vanadium sample.

Measurements for different rotation angles were combined using the HORACE analysis suite ${ }^{45} . S\left(\mathbf{Q}^{\prime}, \omega\right)$ was rotated by $37.8^{\circ}$ about the $Q_{y^{\prime}}$ axis to analyse the data in the $(x, y, z)$ molecular reference frame. The two molecules in the unit cell were included in the calculation of the scattering function and fitting of equation (2) to the $\mathbf{Q}$ dependence of the observed transitions was performed using HORACE. Some compensation for inhomogeneity in the scattering intensity due to self-shielding is performed, based on the variation of incoherent scattering intensity with sample orientation.

Received 18 June 2012; accepted 22 August 2012; published online 30 September 2012

\section{References}

1. Krause, S., Berbil-Bautista, L., Herzog, G., Bode, M. \& Wiesendanger, R. Current-induced magnetization switching with a spin-polarized scanning tunneling microscope. Science 317, 1537-1540 (2007).

2. Mannini, M. et al. Magnetic memory of a single-molecule quantum magnet wired to a gold surface. Nature Mater. 8, 194-197 (2009).

3. Bogani, L. \& Wernsdorfer, W. Molecular spintronics using single-molecule magnets. Nature Mater. 7, 179-186 (2008).

4. Loth, S. et al. Controlling the state of quantum spins with electric currents. Nature Phys. 6, 340-344 (2010).

5. Sanvito, S. Molecular spintronics. Chem. Soc. Rev. 40, 3336-3355 (2011).

6. Khajetoorians, A. A., Wiebe, J., Chilian, B. \& Wiesendanger, R. Realizing all-spin-based logic operations atom by atom. Science 332, 1062-1064 (2011).

7. Candini, A., Klyatskaya, S., Ruben, M., Wernsdorfer, W. \& Affronte, M. Graphene spintronic devices with molecular nanomagnets. Nano Lett. 11, 2634-2639 (2011).

8. Leuenberger, M. N. \& Loss, D. Quantum computing in molecular magnets. Nature 410, 789-793 (2001).

9. Troiani, F. et al. Molecular engineering of antiferromagnetic rings for quantum computation. Phys. Rev. Lett. 94, 207208 (2005).

10. Hirjibehedin, C. F., Lutz, C. P. \& Heinrich, A. J. Spin coupling in engineered atomic structures. Science 312, 1021-1024 (2006).

11. Khajetoorians, A. A. et al. Atom-by-atom engineering and magnetometry of tailored nanomagnets. Nature Phys. 8, 497-503 (2012).

12. Loth, S., Baumann, S., Lutz, C. P., Eigler, D. M. \& Heinrich, A. J. Bistability in atomic-scale antiferromagnets. Science 335, 196-199 (2012). 
13. Gatteschi, D., Sessoli, R. \& Villain, J. Molecular Nanomagnets (Oxford Univ. Press, 2006).

14. Mannini, M. et al. Quantum tunnelling of the magnetization in a monolayer of oriented single-molecule magnets. Nature 468, 417-421 (2010).

15. Corradini, V. et al. Magnetic anisotropy of $\mathrm{Cr}_{7} \mathrm{Ni}$ spin clusters on surfaces. Adv. Funct. Mater. 22, 3706-3713 (2012).

16. Friedman, J. R., Sarachik, M. R., Tejada, J. \& Ziolo, R. Macroscopic measurement of resonant magnetization tunneling in high-spin molecules. Phys. Rev. Lett. 76, 3830-3833 (1996).

17. Thomas, L. et al. Macroscopic quantum tunnelling of magnetization in a single crystal of nanomagnets. Nature 383, 145-148 (1996).

18. Wernsdorfer, W. \& Sessoli, R. Quantum phase interference and parity effects in magnetic molecular clusters. Science 284, 133-135 (1999).

19. Schnack, J. Effects of frustration on magnetic molecules: A survey from Olivier Kahn until today. Dalton Trans. 39, 4677-4686 (2010).

20. Chiolero, A. \& Loss, D. Macroscopic quantum coherence in molecular magnets. Phys. Rev. Lett. 80, 169-172 (1998).

21. Waldmann, O. et al. Quantum phase interference and Néel-vector tunneling in antiferromagnetic molecular wheels. Phys. Rev. Lett. 102, 157202 (2009).

22. Lehmann, J., Gaita-Ario, A., Coronado, E. \& Loss, D. Spin qubits with electrically gated polyoxometalate molecules. Nature Nanotech. 2, 312-317 (2007).

23. Trif, M., Troiani, F., Stepanenko, D. \& Loss, D. Spin-electric coupling in molecular magnets. Phys. Rev. Lett. 101, 217201 (2008).

24. Santini, P., Carretta, S., Troiani, F. \& Amoretti, G. Molecular nanomagnets as quantum simulators. Phys. Rev. Lett. 107, 230502 (2011).

25. Wernsdorfer, W., Aliaga-Alcalde, N., Hendrickson, D. N. \& Christou, G. Exchange-biased quantum tunnelling in a supramolecular dimer of single-molecule magnets. Nature 416, 406-409 (2002).

26. Hill, S., Edwards, R. S., Aliaga-Alcalde, N. \& Christou, G. Quantum coherence in an exchange-coupled dimer of single-molecule magnets. Science 302, 1015-1018 (2003).

27. Timco, G. et al. Engineering the coupling between molecular spin qubits by coordination chemistry. Nature Nanotech. 4, 173-178 (2009).

28. Candini, A. et al. Entanglement in supramolecular spin systems of two weakly coupled antiferromagnetic rings (purple-Cr ${ }_{7} \mathrm{Ni}$ ). Phys. Rev. Lett. 104, 037203 (2010).

29. Ardavan, A. et al. Will spin-relaxation times in molecular magnets permit quantum information processing? Phys. Rev. Lett. 98, 057201 (2007).

30. Schlegel, C., van Slageren, J., Manoli, M., Brechin, E. K. \& Dressel, M. Direct observation of quantum coherence in single-molecule magnets. Phys. Rev. Lett. 101, 147203 (2008)

31. Bertaina, S. et al. Quantum oscillations in a molecular magnet. Nature 453, 203-206 (2008).

32. Wedge, C. J. et al. Chemical engineering of molecular qubits. Phys. Rev. Lett. 108, 107204 (2012)

33. Van Slageren, J. et al. Magnetic anisotropy of the antiferromagnetic ring $\left[\mathrm{Cr}_{8} \mathrm{~F}_{8} \mathrm{Piv}_{16}\right]$. Chem. Eur. J. 8, 277-285 (2002).

34. Santini, P. et al. Spin dynamics and tunneling of the Néel vector in the $\mathrm{Fe}_{10}$ magnetic wheel. Phys. Rev. B 71, 184405 (2005).

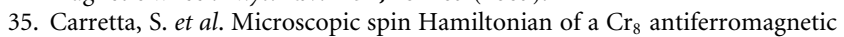
ring from inelastic neutron scattering. Phys. Rev. B 67, 094405 (2003).

36. Meier, F. \& Loss, D. Thermodynamics and spin-tunneling dynamics in ferric wheels with excess spin. Phys. Rev. B 64, 224411 (2001).

37. Carretta, S. et al. Topology and spin dynamics in magnetic molecules. Phys. Rev. B 72, 060403 (2005).

38. Waldmann, O., Guidi, T., Carretta, S., Mondelli, C. \& Dearden, A. L. Elementary excitations in the cyclic molecular nanomagnet $\mathrm{Cr}_{8}$. Phys. Rev. Lett. 91, 237202 (2003).
39. Schnack, J. \& Luban, M. Rotational modes in molecular magnets with antiferromagnetic Heisenberg exchange. Phys. Rev. B 63, 014418 (2000).

40. Waldmann, O. Spin dynamics of finite antiferromagnetic Heisenberg spin rings. Phys. Rev. B 65, 024424 (2001).

41. Waldmann, O. et al. Exchange-coupling constants, spin density map, and $Q$ dependence of the inelastic neutron scattering intensity in single-molecule magnets. Phys. Rev. B 75, 174438 (2007).

42. Marshall, W. \& Lovesey, S. W. Theory of Thermal Neutron Scattering (Oxford Univ. Press, 1971).

43. Ollivier, J. \& Mutka, H. IN5 cold neutron time-of-flight spectrometer, prepared to tackle single crystal spectroscopy. J. Phys. Soc. Jpn 80, SB003 (2011).

44. Bewley, R. J., Taylor, J. W. \& Bennington, S. M. LET, a cold neutron multi-disk chopper spectrometer at ISIS. Nucl. Instrum. Meth. Phys. Res. A 637, 128-134 (2011)

45. Perring, T. G., Ewings, R. A. \& Duijn, J. V. Horace: Visualising and manipulating $S(\mathbf{q}, \omega)$ measured in all four dimensions. http://horace.isis.rl.ac.uk. (2009).

46. Hallberg, K., Wang, X. Q. G., Horsch, P. \& Moreo, A. Critical behavior of the $S=3 / 2$ antiferromagnetic Heisenberg chain. Phys. Rev. Lett. 76, 4955-4958 (1996).

47. Cador, O. et al. The magnetic Möbius strip: Synthesis, structure, and magnetic studies of odd-numbered antiferromagnetically coupled wheels. Angew. Chem. 116, 5308-5312 (2004).

48. Schröder, C. et al. Competing spin phases in geometrically frustrated magnetic molecules. Phys. Rev. Lett. 94, 017205 (2005).

49. Furukawa, Y. et al. Evidence of spin singlet ground state in the frustrated antiferromagnetic ring $\mathrm{Cr}_{8}$ Ni. Phys. Rev. B 79, 134416 (2009).

50. Kenzelmann, M. et al. Structure of end states for a haldane spin chain. Phys. Rev. Lett. 90, 087202 (2003).

51. Gerbeleu, N. V. et al. Octanuclear chromium(III) fluoro-trimethylacetate-a macrocyclic complex. Dokl. Akad. Nauk. SSSR 313, 1459-1462 (1990).

\section{Acknowledgements}

M.L.B. thanks the Japan Society for the Promotion of Science for a postdoctoral fellowship. We acknowledge the Institute Laue-Langevin for financial support and neutron instrument time. We thank the Institute Laue-Langevin technical staff, in particular R. Ammer who designed, machined and made alterations to the sample holders for the present experiments. T.G. thanks R. A. Ewings for the support with the HORACE package. This work was supported by the European Community through the ICT-FET Open Project MolSpinQIP, contract N. 211,284, and by the Progetti di Interesse Nazionale (PRIN) project of the Italian Ministry of Research. R. E. P. W. is supported by a Royal Society Wolfson Merit Award. S.C., G.A. and P.S. are supported by Fondazione Cariparma.

\section{Author contributions}

M.L.B., T.G., S.C., J.O., H.M., H.U.G. and G.A. performed the experiment on a crystal synthesized by G.A.T. after discussion with E.J.L.M. and R.E.P.W. Data treatment was carried out by M.L.B., T.G., J.O. and H.M., and data simulations and fits were performed by M.L.B., T.G. and S.C.; S.C., G.A. and P.S. developed the idea to use four-dimensional INS measurements for a direct extraction of dynamical correlation functions of molecular nanomagnets. S.C., G.A. and P.S. also performed theoretical calculations and wrote the manuscript with input from all co-authors.

\section{Additional information}

Supplementary information is available in the online version of the paper. Reprints and permissions information is available online at www.nature.com/reprints. Correspondence and requests for materials should be addressed to P.S.

\section{Competing financial interests}

The authors declare no competing financial interests. 